\title{
Fatores associados à hipertensão arterial e níveis pressóricos encontrados entre adolescentes trabalhadores*
}

\author{
HYPERTENSION ASSOCIATED FACTORS AND BLOOD PRESSURE AMONG ADOLESCENT \\ WORKERS
}

\section{FACTORES ASOCIADOS A LA HIPERTENSIÓN ARTERIAL Y NIVELES PRESÓRICOS ENCONTRADOS ENTRE ADOLESCENTES TRABAJADORES}

\author{
Carla Spinella ${ }^{1}$, José Luiz Tatagiba Lamas ${ }^{2}$
}

\footnotetext{
* Extraído do trabalho desenvolvido como pesquisa de iniciação científica, com bolsa do Serviço de Apoio ao Estudante da Universidade Estadual de Campinas.

1 Enfermeira.

Enfermaria de Emergências Clínicas e Cirurgia do Trauma do Hospital de Clínicas da Universidade Estadual de Campinas carla.spinella@ terra.com.br.

2 Prof. Ass. Doutor do Departamento de Enfermagem da Faculdade de Ciências Médicas da Universidade Estadual de Campinas zelamas@ fcm.unicamp.br
}

\section{RESUMO}

O objetivo deste trabalho foi identificar fatores associados (FA) à hipertensão arterial e verificar níveis pressóricos de adolescentes trabalhadores. Foram entrevistados 193 adolescentes, sendo 135 homens e 58 mulheres, entre 16 e 18 anos. Após cinco minutos sentados, mediu-se a circunferência braquial e determinou-se a pressão arterial (PA), usando manguito de largura correta (MLC) e padrão (MLP). Os valores obtidos foram relacionados aos FA encontrados. As médias pressóricas, considerando todos os adolescentes, foram $105,2 / 60,9 \mathrm{mmHg}$ (MLC) e 101,0/ 57,9 (MLP-p<0,05). Dentre os FA encontrados, apenas a cor e a ingestão de bebidas alcoólicas foram associadas ao aumento da PA. O uso do MLC permitiu a detecção de maior número de hipertensos e limítrofes que o uso do MLP. Todos apresentaram FA. Pode-se concluir que há vários FA com hipertensão arterial nessa população, alguns deles já causando elevação da PA. Estudos como este deveriam ser realizados freqüentemente entre adolescentes, pois seus níveis pressóricos podem predizer hipertensão na fase adulta.

\section{DESCRITORES}

Hipertensão.

Adolescente.

Fatores de risco.

\begin{abstract}
The aim of this study was to identify hypertension associated factors (AF) and verify blood pressure (BP) levels among adolescent workers. Interviews were conducted with 193 working adolescents - 135 men and 58 women between 16 and 18 years. After five minutes in seated position, their arm circumferences were measured and their BP was determined using correct (CCW) and standard (SCW) cuff widths. The values were related to AF. BP was, in average, $105.2 / 60.9 \mathrm{mmHg}$ (CCW) and 101.0/57.9 (SCW$\mathrm{p}<0.05)$. Among the AF found, only skin color and alcoholic drinking were associated to BP elevation, also noted in male adolescents. When using CCW, more hypertensive and borderline adolescents were found than using SCW. All of them had at least one AF. It can be concluded that there are several hypertension AF among this population, and some of them are already causing BP elevation. Studies such as this should be made frequently because adolescent blood pressure levels can predict hypertension in adulthood.
\end{abstract}

\section{KEY WORDS}

Hypertension.

Adolescent.

Risk factors.

\section{RESUMEN}

El objetivo de nuestra investigación fue identificar factores asociados (FA) con hipertensión arterial y verificar niveles presóricos de adolescentes trabajadores. Fueron entrevistados 193 adolescentes, 135 hombres y 58 mujeres entre 16 y 18 años. Después de cinco minutos sentados, se midieron sus circunferencias braquiales y se determinó sus presiones arteriales (PA) con manguito de ancho correcto (MAC) y estándar (MAP). Los valores observados fueron relacionados a los FA encontrados. Las medias presóricas entre los adolescentes fueron 105,2/60,9 $\mathrm{mmHg}$ (MAC) y 101,0/57,9 (MAP-p<0,05). Entre los FA encontrados, apenas el color de la piel y el consumo de bebidas alcohólicas fueron asociados al aumento de PA. Usando el MAC fueron detectados más hipertensos y limítrofes que usando el MAP. Todos presentaban FA. Se puede concluir que hay diversos FA para hipertensión arterial en esta población, algunos ya causando elevación de la PA. Estudios como éste deberían ser realizados frecuentemente entre adolescentes, porque sus niveles presóricos pueden preceder hipertensión en la fase adulta.

\section{DESCRIPTORES}

Hipertensión.

Adolescente.

Factores de riesgo. 


\section{INTRODUÇÃO}

A hipertensão arterial é uma entidade clínica multifatorial de distribuição não homogênea nas diferentes faixas etárias. Sua incidência entre adolescentes é de 6 a $8 \%{ }^{(1)}$. Os fatores de risco para sua instalação são divididos em: modificáveis, relacionados a hábitos de vida, como sedentarismo ${ }^{(2)}$, ingestão de sal e gordura ${ }^{(1)}$, excesso de peso ${ }^{(2-4)}$, aspectos emocionais como irritação e depressão( ${ }^{(5)}$, uso de drogas ilícitas $^{(1)}$; não modificáveis, relacionados a características herdadas ou inalteráveis, como herança familiar ${ }^{(3,6-8)}$, raça, sexo, idade $^{(1)}$, baixo peso ao nascer ${ }^{(9-11)}$. Todos estes fatores são encontrados nas populações mais jovens. A entrada do adolescente no mercado de trabalho diminui seu tempo disponível para a prática de esportes, acentuando o sedentarismo. Além disso, sua responsabilidade aumenta, trazendo novos componentes que podem causar estresse. Estes dois fatores podem aumentar a exposição dos adolescentes aos fatores de risco para o desenvolvimento de hipertensão arterial.

Devido à pressão sangüínea na infância e adolescência ser considerada um fator prognóstico para a pressão sangüínea adulta ${ }^{(9,12-13)}$, ela deveria ser amplamente pesquisada e mais programas educativos deveriam ser desenvolvidos com esta faixa etária.

O valor da pressão arterial dessa população pode ser fortemente influenciado pelo conhecido efeito da relação entre largura do manguito e circunferência do braço: considerando que se trata de indivíduos em desenvolvimento, é provável que seja encontrado um grande número de pessoas com braços finos, situação em que o uso do manguito padrão pode fornecer valores baixos quando a pressão arterial é normal e normais quando a pressão arterial é elevada ${ }^{(14)}$.

Tendo em vista a indisponibilidade de manguitos de diferentes larguras na quase totalidade dos serviços de saúde, torna-se muito provável que em adolescentes ocorra subestimação da pressão arterial. Se for considerada a população de adolescentes trabalhadores, provavelmente expostos aos fatores de risco já mencionados, é importante a determinação da pressão arterial com manguitos de largura correta e a busca ativa de fatores associados com hipertensão arterial, visando a detecção precoce de níveis pressóricos elevados, que possibilita um tratamento rápido e diminuição de seus efeitos deletérios.

\section{OBJETIVOS}

a) Identificar fatores associados com hipertensão arterial em adolescentes que trabalham na Universidade Estadual de Campinas (UNICAMP).

b) Relacionar os fatores associados à hipertensão aos níveis pressóricos observados.

\section{MÉTODO}

Trata-se de estudo transversal, realizado com a população de adolescentes exercendo a função de mensageiros na Universidade Estadual de Campinas.

A coleta de dados foi realizada por meio de um instrumento desenvolvido pelos autores, com dados de identificação, fatores associados com hipertensão arterial relatados pelos adolescentes e espaço para anotação de seus valores de pressão arterial. A pesquisa teve início, após a aprovação do Comitê de Ética em Pesquisa da Faculdade de Ciências Médicas da UNICAMP, com a realização do préteste do instrumento, do qual participaram 20 adolescentes com as mesmas características daqueles que comporiam a população do estudo. Um termo de consentimento livre e esclarecido foi assinado pelo adolescente e seu responsável, em duas vias, sendo uma via para ele e outra para o pesquisador. Os entrevistados não conseguiram responder algumas perguntas por desconhecer as respostas. Estas perguntas foram impressas no verso da cópia do termo de consentimento que seria devolvido ao pesquisador. Assim, quando o responsável o assinasse, poderia também respondê-las.

No início da pesquisa foi feito um contato prévio com os setores da universidade responsáveis pela contratação de mensageiros, com a finalidade de obter uma lista contendo seus nomes, locais de trabalho e telefones. O número inicial de mensageiros era de 203.

Para a coleta de dados, foi feito um agendamento por contato telefônico com cada mensageiro e sua chefia. Cada adolescente permaneceu sentado durante pelo menos 5 minutos, antes do início das medidas de pressão arterial. Nesse intervalo eles responderam o questionário para levantamento de fatores associados com hipertensão arterial, sendo que a estatura e o peso eram indicados pelo adolescente. Para as variáveis irritação, estresse e raiva, foi considerada a opinião dos adolescentes sobre os fatores que as provocavam em seu dia a dia. Quando eles manifestavam dúvida quanto à conceituação desses termos, definíamos irritação como uma sensação de nervosismo mais passageira, estresse como uma situação permanente relacionada com cansaço e sensação de insatisfação e raiva como uma situação de forte irritação, com duração também mais prolongada.

Uma única observadora treinada mediu a pressão arterial dos participantes, usando a técnica proposta pela American Heart Association ${ }^{(15)}$. A circunferência do braço foi medida no ponto mais largo do bíceps, de acordo com o protocolo instituído por Arcuri(16). O braço esquerdo foi mantido estendido e apoiado, no nível do $4^{\circ}$ espaço intercostal. Foram realizadas três mensurações da pressão arterial, utilizandose um manômetro aneróide e a campânula de um estetoscópio para adultos. As duas primeiras medidas foram feitas usan- 
do o manguito de largura correta (38\% da circunferência do braço) e a última com o manguito padrão $(12 \mathrm{~cm})$. As larguras de manguito disponíveis foram $8,9,12,15$ e 18 centímetros, as únicas encontradas no comércio após exaustiva busca. Entretanto, o manguito de 18 centímetros não foi utilizado. As circunferências de braço abrangidas por cada manguito foram, em centímetros, 20 a 22 (para o manguito 8), 23 a 27 (para o manguito 9), 28 a 36 (para o manguito 12) e 37 a 44 (para o manguito 15). A pressão sistólica foi determinada no momento do aparecimento do $1^{\circ}$ ruído (Fase I de Korotkoff) e a pressão diastólica no desaparecimento dos sons (Fase V de Korotkoff); quando os ruídos persistiram até próximo ao nível zero do manômetro, a pressão diastólica foi determinada no momento do abafamento dos sons (fase IV de Korotkoff). Os valores pressóricos obtidos foram definidos como elevados ou normais de acordo com as IV Diretrizes Brasileiras de Hipertensão Arterial ${ }^{(17)}$. A primeira medida não foi considerada para análise estatística, já que poderia apresentar-se alterada em razão de não ter ocorrido ainda habituação dos sujeitos ao procedimento ${ }^{(18)}$.

Os dados foram organizados em planilhas no programa Microsoft Excel e submetidos à análise estatística. Para verificar associação ou comparar proporções foram utilizados o teste Qui-Quadrado ou o teste Exato de Fisher. Para comparação das variáveis contínuas entre as classes de variáveis com duas categorias foi utilizado o teste $t$ de Student, quando a distribuição da variável apresentou normalidade, e o teste de Mann-Whitney, caso contrário. Para comparação das variáveis contínuas entre as classes de variáveis com três categorias foi utilizada a Análise de Variância, quando a distribuição da variável apresentou normalidade, e o teste de Kruskal-Wallis, caso contrário. Para verificar a concordância das duas avaliações das pressões foram utilizados a Análise de Regressão Linear Simples e o coeficiente de correlação de Pearson. Foram considerados significativos valores de $\mathrm{p}<0,05^{(19-20)}$.

\section{RESULTADOS E DISCUSSÃO}

A população final constou de 193 adolescentes, sendo 58 do sexo feminino e 135 do sexo masculino; a faixa etária oscilou de 16 anos completos a 18 anos incompletos e a escolaridade variou da oitava série do ensino fundamental à primeira série do ensino superior. Quanto à cor da pele, encontrou-se 79 adolescentes brancos, 52 negros e 62 pardos.

A Tabela 1 apresenta outras características biológicas dos adolescentes. Observa-se que o valor médio do Índice de Massa Corpórea (IMC) ${ }^{(21)}$ está dentro da normalidade. No entanto, alguns indivíduos apresentaram obesidade e outros mostraram-se muito magros, como pode ser evidenciado pelos valores máximo e mínimo deste parâmetro. O valor médio das circunferências de braço indica que, entre os manguitos disponíveis, o mais adequado para a amostra estudada foi o de nove centímetros de largura, resultado que pode ser confirmado pela análise do Gráfico 1. Os valores máximo e mínimo deste parâmetro situam a faixa de larguras de manguito necessária para estes adolescentes entre oito e 15 centímetros.

Tabela 1 - Valores médios, máximo e mínimo de estatura, índice de massa corpórea (IMC) e circunferência do braço (CB) dos adolescentes - Campinas - 2003

\begin{tabular}{lccc}
\hline & Estatura & IMC & CB \\
\hline Média & $\begin{array}{c}1,69 \\
(0,08)\end{array}$ & $\begin{array}{c}21,42 \\
(3,05)\end{array}$ & $\begin{array}{r}26,05 \\
(3,01)\end{array}$ \\
\hline V. máx. & 1,88 & 35,41 & 39,00 \\
\hline V. min. & 1,46 & 13,96 & 20,60 \\
\hline $\mathbf{N}$ & $\mathbf{1 , 8 8}^{*}$ & $\mathbf{1 6 4}^{*}$ & $\mathbf{1 9 3}$ \\
\hline
\end{tabular}

*Casos incompletos devido à falta de indicação do peso e/ou estatura Desvio padrão indicado entre parênteses

Observa-se no Gráfico 1 que o manguito mais utilizado foi o de $9 \mathrm{~cm}$ de largura, seguido do manguito padrão (12 $\mathrm{cm}$ ). Ainda, se pode notar que $77,4 \%$ da população utilizaram manguitos menores do que o padrão. Este resultado é previsível em função do IMC e da circunferência de braço encontrados (Tabela 1). Além disso, está de acordo com dados da literatura nacional.

Gráfico 1 - Freqüência absoluta e percentual de utilização das diferentes larguras de manguito - Campinas - 2003

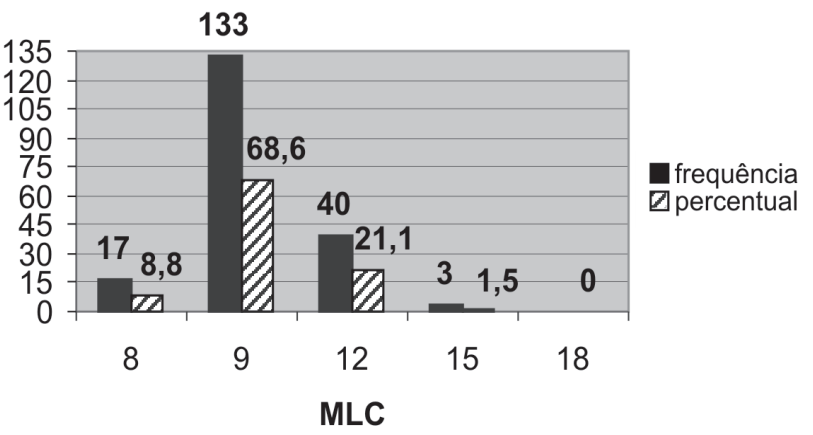

Em um estudo ${ }^{(15)}$, constatou-se que 93,2\% dos indivíduos da população estudada utilizaram manguitos menores do que o de largura padrão. Outros pesquisadores ${ }^{(22)}$ relataram que $80,5 \%$ das gestantes abordadas em seus estudos utilizaram manguitos menores que o padrão.

As diferenças encontradas entre nossos resultados e os de outros autores ${ }^{(16-22)}$, podem estar relacionadas às peculiaridades de cada população. Além disso, no atual estudo foram usados manguitos de largura 8, 9, 12 e $15 \mathrm{~cm}$, enquanto os autores citados utilizaram manguitos com largura variando de $1 \mathrm{em} 1 \mathrm{~cm}$, a partir de $8 \mathrm{~cm}$. É possível que, em nossa população, alguns usuários do manguito de $12 \mathrm{~cm}$ devessem utilizar um com largura de $11 \mathrm{~cm}$, fato que aproximaria nosso percentual dos resultados encontrados por aqueles 
autores. Em outro estudo ${ }^{(18)}, 75,4 \%$ dos sujeitos utilizaram manguitos mais estreitos do que 12 centímetros, mas se tratava de uma população composta predominantemente por cardiopatas obesos.

O resultado da utilização do manguito com $15 \mathrm{~cm}$ de largura é previsível em função do IMC agrupado (Tabela 2): a maior parte dos mensageiros apresentou IMC que permitiu situá-los nas faixas de normalidade e baixo peso. As faixas de sobrepeso e obesidade graus I e II corresponderam à minoria em nosso estudo atingindo, somadas, 8,5\% dos participantes do estudo.

Tabela 2 - Freqüência absoluta e percentual de IMC agrupado - Campinas - 2003

\begin{tabular}{lrr}
\hline Classificação & N & \% \\
\hline Abaixo do peso & 22 & 13,4 \\
Normal & 128 & 78,0 \\
Sobrepeso & 11 & 6,7 \\
Obesidade grau I & 2 & 1,2 \\
Obesidade grau II & 1 & 0,6 \\
\hline Total & 164 & 100,0 \\
\hline
\end{tabular}

Não houve significância estatística com relação à influência da faixa de IMC na pressão arterial dos sujeitos entrevistados.

Oitenta e oito adolescentes informaram o peso ao nascimento. Destes, 13 apresentaram baixo peso ao nascer ${ }^{(23)}$. Não foi encontrada relação significativa entre os valores de peso ao nascimento e os níveis de pressão arterial sistólica e diastólica aferidos com manguitos de largura correta e pa- drão ( $p>0,05$ - teste de Kruskal-Wallis). Segundo outros trabalhos ${ }^{(9,24)}$, essa relação também não foi significativa. Alguns autores mostraram relação significativa e inversamente proporcional entre baixo peso ao nascer e pressão arterial sistólica, que apresentou-se elevada ${ }^{(25)}$.

As médias de circunferência de braço entre os grupos de adolescentes que apresentaram baixo e alto peso ao nascer diferiram significativamente, sendo mais alta no primeiro grupo $[27,73 \mathrm{~cm} \pm 3,12$ e $25,42 \pm 3,03$, respectivamente $(\mathrm{p}=0,0112$ - teste de Kruskal-Wallis)]. Quando se relacionou o peso ao nascer com o IMC atual, foi encontrada uma tendência maior ( $\mathrm{p}=$ 0,0755 - teste exato de Fisher) dos adolescentes que nasceram com baixo peso desenvolverem sobrepeso $(33,3 \%)$, se comparados àqueles que nasceram com peso adequado $(21,0 \%)$ ou alto $(4,65 \%)$. Alguns pesquisadores já conseguiram demonstrar essa relação de forma significativa ${ }^{(26)}$.

Na Tabela 3 encontram-se os dados relacionados aos fatores modificáveis. Pelo percentual referido do uso de substâncias, especialmente drogas ilícitas e tabaco, é possível que alguns tenham omitido tais informações por timidez ou medo, já que é sabido que o uso e abuso de drogas constitui um dos mais graves problemas enfrentados pela sociedade contemporânea. Dados do Centro Brasileiro de Informações Sobre Drogas Psicotrópicas (CEBRID) revelaram, em 1997, que $51,2 \%$ dos adolescentes entre 10 e 12 anos já havia usado álcool; $11 \%$, tabaco; 7,8\%, solventes; $2 \%$, ansiolíticos; $1,8 \%$, anfetaminas ${ }^{(27)}$.

Nota-se um grande percentual de sedentarismo. Os adolescentes alegam não ter mais tempo para práticas esportivas, já que o trabalho envolve todo o período diurno e estudam à noite.

Tabela 3 - Freqüência absoluta e percentual de fatores associados modificáveis encontrados na população - Campinas - 2003

\begin{tabular}{lcc}
\hline Fatores encontrados $^{*}$ & N & \% \\
\hline Irritação & 120 & 62,6 \\
Sedentarismo & 118 & 60,8 \\
Ingestão de cafeína & 112 & 57,7 \\
Estresse & 109 & 56,9 \\
Raiva & 104 & 54,0 \\
Ingestão de bebida alcoólica & 56 & 28,9 \\
Uso de anticoncepcionais hormonais ${ }^{* *}$ & 14 & 24,1 \\
Uso exagerado de sal & 27 & 13,9 \\
Tabagismo & 6 & 3,1 \\
Drogas ilícitas & - & - \\
\hline
\end{tabular}

\footnotetext{
*cada adolescente referiu um fator associado ou mais
}

**pergunta feita às participantes do sexo feminino 
Pode-se observar também a elevada frequiência (acima de 50\%) de estresse, irritação e raiva. Os adolescentes apontaram como suas causas o trabalho e a família, na maior parte das vezes.

Os adolescentes relataram ter livre acesso ao café feito em seu setor, o que justifica o alto consumo de cafeína.
Também relacionaram o aumento do consumo ao início de suas atividades na universidade.

Nenhum fator de risco exerceu influência sobre os valores de pressão arterial diastólica; por outro lado, apenas o consumo de álcool, raiva e sedentarismo foram capazes de influenciar a pressão sistólica verificada (Tabela 4).

Tabela 4 - Fatores associados à hipertensão e médias de pressão arterial sistólica em função da largura do manguito Campinas - 2003

\begin{tabular}{llrr}
\hline Fatores associados & & MLC $^{1}$ & MLP $^{2}$ \\
\hline Raiva & Presente & $104,0^{*}(11,0)$ & $93,0(7,0)$ \\
& Ausente & $107,0(13,0)$ & $93,0(9,0)$ \\
Sedentarismo & Presente & $104,0^{*}(12,0)$ & $100,0(12,0)$ \\
& Ausente & $108,0(12,0)$ & $103,0(11,0)$ \\
Ingestão de bebida alcoólica & Presente & $109,0^{*}(12,3)$ & $105,7^{*}(11,4)$ \\
& Ausente & $103,7(11,6)$ & $99,3(11,4)$ \\
\hline
\end{tabular}

* diferença significativa em relação ao grupo ausente $(\mathrm{p}<0,05$ - Teste $\mathrm{t}$ de Student)

$1 \mathrm{MLC}=$ manguito de largura correta

2 MLP = manguito de largura padrão

Desvio padrão indicado entre parênteses

É surpreendente notar que na ausência de raiva e sedentarismo a média de pressão arterial sistólica obtida com o manguito correto foi maior do que na presença destes fatores. Isso contradiz o que seria esperado, no caso do sedentarismo.
Foi observada baixa freqüência de antecedentes pessoais para algumas doenças que são fatores associados não modificáveis para hipertensão arterial; já a ocorrência familiar das mesmas foi elevada. Entretanto, em nenhuma das situações eles alteraram os valores pressóricos (Tabela 5).

Tabela 5 - Freqüência absoluta e percentual de fatores associados não modificáveis, pessoal e familiar - Campinas - 2003

\begin{tabular}{|c|c|c|c|c|c|c|}
\hline \multirow[t]{2}{*}{ Fatores associados } & \multicolumn{3}{|c|}{ Pessoal } & \multicolumn{3}{|c|}{ Familiar } \\
\hline & $\mathbf{N}$ & $\mathbf{n}$ & $\%$ & $\mathbf{N}$ & $\mathrm{n}$ & $\%$ \\
\hline Diabetes Mellitus & 193 & 1 & 0,5 & 110 & 64 & 58,2 \\
\hline Doença Cardiovascular & 193 & 3 & 1,6 & 101 & 46 & 45,5 \\
\hline Hipertensão Arterial & 193 & 1 & 0,5 & 103 & 65 & 63,1 \\
\hline Doença Renal & 193 & 1 & 0,5 & 101 & 21 & 20,8 \\
\hline
\end{tabular}

Na Tabela 6 são apresentadas as médias da pressão arterial em função da cor da pele e da largura do manguito.

Tabela 6 - Médias de pressão arterial em função da largura do manguito e da cor da pele - Campinas - 2003

\begin{tabular}{llrr}
\hline Cor da pele & PA & MLC & MLP $^{\mathbf{2}}$ \\
\hline \multirow{2}{*}{ Branco } & Sistólica & $107,9^{*}(12,3)$ & $102,7(12,4)$ \\
& Diastólica & $62,5(9,8)$ & $59,3(9,4)$ \\
Negro & Sistólica & $105,2(10,8)$ & $100,1(11,4)$ \\
& Diastólica & $60,2(7,7)$ & $57,5(9,2)$ \\
Pardo & Sistólica & $101,9(12,0)$ & $100,0(11,1)$ \\
& Diastólica & $59,8(8,8)$ & $56,8(6,9)$ \\
\hline
\end{tabular}

* diferença significativa entre brancos e pardos $(\mathrm{p}=0,0136$ - ANOVA)

1 MLC = manguito de largura correta

$2 \mathrm{MLP}=$ manguito de largura padrão

Desvio padrão indicado entre parênteses 
A média de pressão arterial sistólica encontrada entre os brancos foi maior, atingindo níveis de significância quando comparada com a média entre os pardos. Este achado, assim como aquele relacionado ao sedentarismo (Tabela 4), contradiz o que se esperava.

Nos Gráficos 2 e 3 são apresentados os valores médios (com desvio-padrão), máximo e mínimo de pressão arterial obtidos na população. Pode-se observar que houve subestimação das médias dos valores de pressão arterial com o manguito padrão. Apesar das diferenças entre as médias serem de aproximadamente $4 \mathrm{mmHg}$ na pressão sistólica e $3 \mathrm{mmHg}$ na pressão diastólica, a análise dos dados relativos a cada indivíduo revela que esta diferença foi, com freqüência, maior, chegando a superar $10 \mathrm{mmHg}$. Isto pode contribuir para dificultar a detecção precoce de casos de hipertensão e, por conseguinte, retardar o início de um tratamento adequado.

A Tabela 7 apresenta os valores de pressão sistólica e diastólica obtidos com os manguitos de largura correta e padrão em função do sexo.

Gráfico 2 - Média, Desvio-Padrão (DP), Valor Máximo (Max.) e Valor Mínimo (Min.) de pressão arterial sistólica obtidos com manguitos de largura correta e padrão - Campinas - 2003

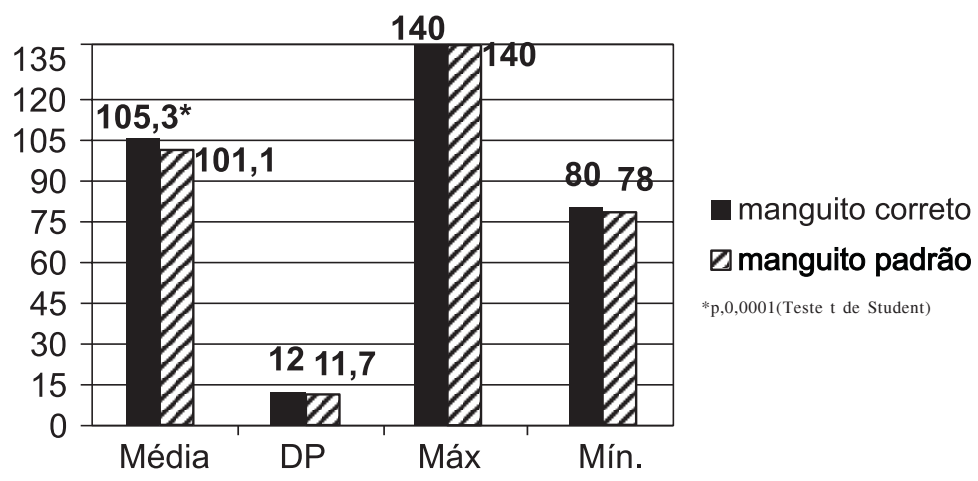

Gráfico 3 - Média, Desvio-Padrão (DP), Valor Máximo (Max.) e Valor Mínimo (Min.) de pressão arterial diastólica obtidos com manguitos de largura correta e padrão - Campinas - 2003

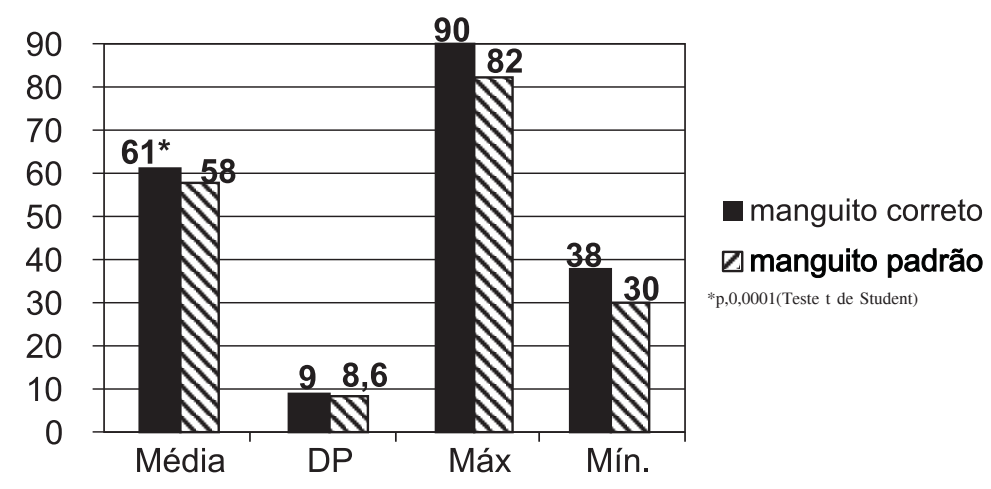

Tabela 7 - Valores médios, máximo e mínimo de pressão arterial sistólica (PAS) e diastólica (PAD) obtidos com os manguitos de largura correta (MLC) e padrão (MLP), em função do sexo - Campinas - 2003

\begin{tabular}{|c|c|c|c|c|c|c|c|}
\hline & & \multicolumn{3}{|c|}{ MLC } & \multicolumn{3}{|c|}{ MLP } \\
\hline & & Média & Máx. & Min. & Média & Máx. & Min. \\
\hline \multirow[t]{2}{*}{ PAS } & Feminino & $97,6^{*}(9,0)$ & 120 & 80 & $93,31^{*}(8,2)$ & 114 & 78 \\
\hline & Masculino & $108,5(11,7)$ & 140 & 80 & $104,44(8,7)$ & 140 & 78 \\
\hline \multirow[t]{2}{*}{ PAD } & Feminino & $58,7^{*}(8,9)$ & 80 & 40 & $57,1(8,8)$ & 82 & 30 \\
\hline & Masculino & $62,0(8,9)$ & 90 & 38 & $58,4(8,6)$ & 80 & 40 \\
\hline
\end{tabular}

*diferença significativa em relação ao sexo masculino $(\mathrm{p}<0,05$ - Teste $\mathrm{t}$ de Student)

Desvio padrão indicado entre parênteses 
Além da subestimação introduzida na medida indireta pelo manguito de largura padrão, já comentada anteriormente, nota-se que os valores pressóricos encontrados no sexo feminino foram persistentemente menores que aqueles observados no sexo masculino, com diferença estatística para as duas larguras de manguito utilizadas. Isto só não foi observado quando foram comparadas as pressões diastólicas medidas com o manguito padrão.

Em função dos menores valores de pressão para o sexo feminino, decidiu-se verificar a proporção de mulheres na população, buscando explicar os resultados inesperados encontrados nas Tabelas 4 e 6 . No que diz respeito à raiva, a parcela da população em que este fator esteve ausente representou $21 \%$ de mulheres, enquanto na parcela que referiu este sentimento esta proporção foi de $36 \%$. No caso do sedentarismo observou-se um comportamento similar: $11,5 \%$ de mulheres na parcela não sedentária e 40,3\% na sedentária, que apresentou menores valores de pressão arterial. No que se refere à cor da pele, mais uma vez a proporção de mulheres foi menor no grupo que apresentou pressão mais alta e maior naquele com médias mais baixas: $22 \%$ entre os brancos, $31 \%$ entre os negros e $39 \%$ entre os pardos. Assim, supõe-se que esses dados poderiam ter sido influenciados pela dife- rente proporção de sujeitos do sexo feminino nestes grupos, cujos menores valores de pressão arterial podem ter provocado as discrepâncias observadas. Seria importante a realização de estudos similares, em que a proporção entre indivíduos de ambos os sexos não fosse tão desigual, para tentar esclarecer melhor estes achados.

Apenas 10 participantes $(5,2 \%)$ tiveram suas pressões classificadas como limítrofe ou elevada, de acordo com as IV Diretrizes Brasileiras de Hipertensão Arterial ${ }^{(17)}$. Isto pode estar relacionado à idade, já que a hipertensão arterial ainda é mais comum a partir da quarta década de vida.

Uma outra classificação importante é aquela apresentada pelo comitê do National High Blood Pressure Education Program Working Group on High Blood Pressure in Children and Adolescents, assumida pela American Academy of Pediatrics (no.). Enquanto as diretrizes nacionais apontam uma faixa limítrofe e outra correspondente a hipertensão, o comitê americano refere-se a estágios pré-hipertensivo (correspondente ao limítrofe), hipertensão 1 e hipertensão 2 (correspondentes ao estágio de hipertensão). No Quadro 1 são apresentados os valores pressóricos dos dez adolescentes que apresentaram pressão arterial elevada e a classificação de cada um de acordo com as duas entidades citadas.

Quadro 1 - Valores pressóricos dos adolescentes com pressão arterial elevada, classificados de acordo com as IV Diretrizes Brasileiras de Hipertensão Arterial (DBHA) e com a American Academy of Pediatrics (AAP), em função da largura do manguito

\begin{tabular}{|c|c|c|c|}
\hline Sujeito & MLC & DBHA & AAP \\
\hline 11 & $136 / 70$ & L/N & PH/N \\
\hline 17 & $118 / 80$ & N/N & N/PH \\
\hline 37 & $126 / 90$ & N/H & N/H1 \\
\hline 55 & $128 / 72$ & L/N & PH/N \\
\hline 57 & $130 / 60$ & L/N & H1/N \\
\hline 65 & $134 / 80$ & H/N & H1/PH \\
\hline 106 & $140 / 62$ & H/N & H1/N \\
\hline 148 & $124 / 84$ & N/L & N/PH \\
\hline 167 & $130 / 60$ & L/N & PH/N \\
\hline 179 & $130 / 48$ & L/N & PH/N \\
\hline
\end{tabular}

\begin{tabular}{|c|c|c|}
\hline MLP & DBHA & AAP \\
\hline $112 / 72$ & $\mathrm{~N} / \mathrm{N}$ & $\mathrm{N} / \mathrm{N}$ \\
\hline $114 / 82$ & $\mathrm{~N} / \mathrm{N}$ & $\mathrm{N} / \mathrm{PH}$ \\
\hline $112 / 80$ & $\mathrm{~N} / \mathrm{N}$ & $\mathrm{N} / \mathrm{N}$ \\
\hline $126 / 72$ & $\mathrm{~L} / \mathrm{N}$ & $\mathrm{PH} / \mathrm{N}$ \\
\hline $130 / 64$ & $\mathrm{~L} / \mathrm{N}$ & $\mathrm{H} 1 / \mathrm{N}$ \\
\hline $124 / 66$ & $\mathrm{~N} / \mathrm{N}$ & $\mathrm{N} / \mathrm{N}$ \\
\hline $140 / 52$ & $\mathrm{H} / \mathrm{N}$ & $\mathrm{H} 1 / \mathrm{N}$ \\
\hline $100 / 76$ & $\mathrm{~N} / \mathrm{N}$ & $\mathrm{N} / \mathrm{N}$ \\
\hline $118 / 60$ & $\mathrm{~N} / \mathrm{N}$ & $\mathrm{N} / \mathrm{N}$ \\
\hline $128 / 50$ & $\mathrm{~N} / \mathrm{N}$ & $\mathrm{PH} / \mathrm{N}$ \\
\hline
\end{tabular}

MLC = manguito correto; MLP = manguito padrão; $\mathrm{N}=$ normal; $\mathrm{L}=$ limítrofe; $\mathrm{H}=$ hipertensão arterial; $\mathrm{PH}=$ pré-hipertensão; $\mathrm{H} 1$ = hipertensão arterial estágio 1 
Considerando-se a classificação adotada pelas IV Diretrizes Brasileiras de Hipertensão Arterial, 90\% dos indivíduos apresentaram um dos valores (sistólico ou diastólico) nas faixas limítrofe ou de hipertensão quando sua pressão arterial foi medida com o manguito correto. Já com o manguito padrão, apenas $40 \%$ de valores elevados foram detectados, sendo um deles (indivíduo 17) diferente do valor obtido com o manguito correto em apenas $2 \mathrm{mmHg}$, que fizeram com que a classificação fosse alterada. Utilizando o critério adotado pela American Academy of Pediatrics (no.), todos os indivíduos apresentaram um dos valores elevado quando se usou o manguito correto. $O$ manguito padrão só permitiu a detecção de $50 \%$ de anormalidades. Qualquer que seja o critério adotado, percebe-se a grande influência da largura do manguito na identificação de valores elevados de pressão arterial, havendo maior dificuldade de detectá-los quando se usa o manguito padrão.

Este fato é preocupante e foi estudado de maneira especial por uma autora ${ }^{(16)}$. Esta autora constatou que em uma população de 900 pessoas apenas 1,3\% dos magros apresentaram pressão diastólica maior que $89 \mathrm{mmHg}$ quando se usou o manguito de largura padrão, índice que aumentou para 17,6\% com o manguito correto (aumento de 13,5 vezes). Apesar de não serem conclusivos devido à baixa ocorrência, dois indivíduos em nossa população apresentaram resultados similares: de acordo com a classificação utilizada, eles foram considerados hipertensos sistólico ou diastólico quando sua pressão foi medida com o manguito correto $(9 \mathrm{~cm})$ e normotensos quando se usou o manguito padrão (Quadro 1, indivíduos 37 e 65). Estes indivíduos apresentaram, respectivamente, IMC de 21,38 e 19,05, valores considerados normais ${ }^{(21)}$. Chama-nos também a atenção o fato de que o segundo adolescente apresentou baixo peso ao nascimento $(1,8 \mathrm{Kg})$, o que vem sendo considerado fator de risco para hipertensão arterial ${ }^{(2,13-14)}$.

Nossa preocupação com a maior detecção de hipertensos propiciada pelo uso do manguito correto se deve ao fato de que apenas o manguito padrão é utilizado rotineiramente. Assim, deixa de se detectar a hipertensão em pessoas cujas circunferências de braço não são adequadas ao manguito de largura padrão. Todos estes adolescentes referiram a presença de mais de um fator associado à hipertensão, exceto o sujeito 37, que só referiu o antecedente familiar. Os outros cinco apresentaram fatores de risco classificados como modificáveis e não modificáveis.

\section{REFERÊNCIAS}

1. Sociedade Brasileira de Hipertensão. Sociedade Brasileira de Cardiologia. Sociedade Brasileira de Nefrologia. III Consenso Brasileiro de Hipertensão Arterial. Campos do Jordão; 1998.

2. Kebede D, Ketsela T. Precursors of atherosclerotic and hypertensive diseases among adolescents in Addis Ababa, Ethiopia. Bull World Health Org. 1994;71(6):787-94.
Todos esses participantes eram homens magros, utilizando o manguito de $9 \mathrm{~cm}$. A dificuldade de detectar níveis pressóricos elevados entre pessoas com essas características é maior entre pessoas obesas e adquire grande importância quando se considera que valores elevados de pressão arterial nesta faixa etária podem ser preditivos de hipertensão arterial entre adultos ${ }^{(2-4)}$. Assim, talvez seja possível considerar que o uso do manguito padrão em indivíduos magros seja um fator de risco para o desenvolvimento de hipertensão arterial.

Não é usual haver jogos de manguitos com diferentes larguras nos serviços de saúde. É provável que isto ocorra por não se considerar necessário o uso deste equipamento, que seria um custo adicional para as instituições. Porém nossos dados reforçam a importância do uso do manguito correto para a identificação precoce de hipertensão arterial, o que seria altamente desejável, já que implicaria em melhor controle desta doença, diminuição das despesas com seu tratamento e redução da prevalência de outras doenças para as quais ela é fator de risco.

\section{CONCLUSÕES}

Os dados obtidos permitem concluir que há diversos fatores associados à hipertensão já presentes entre estes adolescentes. Podem ser destacadas a ingestão de bebidas alcoólicas e a cor como os únicos capazes, em nosso estudo, de aumentar os valores pressóricos. O sexo masculino também atuou como fator associado, já que a pressão arterial dos homens foi significativamente maior do que a das mulheres. Outros fatores, tanto modificáveis como não modificáveis, foram encontrados em mais da metade da população: irritação, sedentarismo, ingestão de cafeína, estresse, raiva e antecedentes familiares para hipertensão arterial e diabete. Também se pode concluir a importância de se medir a pressão corretamente, utilizando manguitos de largura adequada a cada braço, pois as diferenças observadas entre os valores obtidos com as duas larguras freqüentemente superou $10 \mathrm{mmHg}$, quando comparou-se as médias, atingindo significância estatística. Além disso, o uso do manguito de largura correta possibilitou a detecção de maior número de adolescentes com valores de pressão arterial altos ou limítrofes. $\mathrm{O}$ acompanhamento desses adolescentes pode ajudar a definir com maior clareza a influência destes e de outros fatores sobre seus níveis de pressão arterial.

3. Falkner B, Kushner H, Onesti G, Angelakos ET. Cardiovascular characteristics in adolescents who develop essential hypertension. Hypertension. 1981;3(5):521-7.

4. Tanji JL. Tracking of elevated blood pressure values in adolescent athletes at 1-year follow-up. Am J Dis Child. 1991;145(6):665-7. 
5. Ewart CK, Kolodner KB. Negative affect, gender and expressive style predict elevated ambulatory blood pressure in adolescents. J Pers Soc Psychol. 1994;66(3):596-605.

6. Matta M, Rocha JA. Fatores de risco de doença vascular aterosclerótica em crianças e adolescentes. J Pediatr. 1991;67(5/ 6):168-73.

7. Singh BM, Vashisht S, Bachani D. Variations in blood pressure of adolescents in relation to sex and social factors in a rural area of Haryana. Ind J Public Health. 1994;38(1):14-7.

8. Cavalcante JWS, Cavalcante LP, Pacheco WS, Menezes MGF, Gama FCG. Comportamento da pressão arterial em filhos de normotensos e filhos de hipertensos submetidos a estímulos pressóricos. Arq Bras Cardiol. 1997;69(5):323-6.

9. Barros FC, Victoria CG. Increased blood-pressure inadolescents who were small for gestational age at birth: a cohort study in Brazil. Int J Epidemiol. 1999;28(4):676-81.

10. Eriksson J, Forsén T, Tuomilehto J, Osmond C, Barker D. Fetal and childhood growth and hypertension in adult life. Hypertension. 2000;36(5):790-94.

11. Lurbe E, Torro I, Rodríguez C, Alvarez V, Redón J. Birth weight influences blood pressure values and variability in children and adolescents. Hypertension. 2001;38(3):389-93.

12. Nelson MJ, Ragland DR, Syme SL. Longitudinal prediction of adult blood pressure from juvenile blood pressure levels. Am J Epidemiol. 1992;136(6):633-45.

13. Parker FC, Croft JB, Cresanta JL, Freedman DS, Burke GL, Webber LS, et al. The association between cardiovascular response tasks and future blood pressure levels in children: Bogalusa Heart Study. Am Heart J. 1987;113(5):1174-9.

14. Araújo TL, Arcuri EAM, Martins E. Instrumentação na medida da pressão arterial: aspectos históricos, conceituais e fontes de erro. Rev Esc Enferm USP. 1998;32(1):33-41.

15. Perloff D, Grim C, Flack J, Frohlich ED, Hill M, McDonald $\mathrm{M}$, et al. Human blood pressure determination by sphygmomanometry. Circulation. 1993;88(5 Pt 1):2460-70.

16. Arcuri EAM. Estudo comparativo da medida indireta da pressão arterial com manguito de largura correta e com manguito de largura padrão [tese]. São Paulo: Instituto de Ciências Biomédicas, Universidade de São Paulo; 1985.
17. Sociedade Brasileira de Hipertensão. Sociedade Brasileira de Cardiologia. Sociedade Brasileira de Nefrologia. IV Diretrizes Brasileiras de Hipertensão Arterial. Campos do Jordão; 2002.

18. Lamas JLT. Medida da pressão arterial em locais alternativos: comparação de valores diretos e indiretos em função da largura do manguito [tese]. São Paulo: Escola de Enfermagem, Universidade de São Paulo; 1999

19. Agresti A, Finlay B. Statistical methods for the social sciences. San Francisco: Dellen Publishing; 1986.

20. Fleiss JL. Statistical methods for rates and proportions. $2^{\text {nd }}$ ed. New York: Jonh Wiley \& Sons; 1981.

21. World Health Organization (WHO). Obesity: preventing and managing the global epidemic. Report of a WHO consultation of obesity. Geneva; 1997.

22. Oliveira SMJV, Arcuri EAM, Santos JLF. Cuff width influence on blood pressure measurement during the pregnant-puerperal cycle. J Adv Nurs. 2002;38(2):180-9.

23. Gutierrez MT, Pistelli IP, Sartori MEA, Paccez JD, Mori H, Amaral LC. Pediatria: diagnóstico e terapêutica. $2^{\text {a }}$ ed. São Paulo: Robe; 1999.

24. Pearce MS, Sullivan JJO. Relationship between birth weight and blood pressure variability in children. J Hum Hypertens. 2003;17(10):677-80.

25. Gunnarsdottir I, Birgisdottir BE, Benediktsson R, Gudnason V, Thorsdottir I. Relationship between size at birth and hypertension in a genetically homogenous populations of high birth weight. J Hypertens. 2002;20(4);623-8.

26. Ramirez EM, Montero AG, Del Sol IMM, Paneque RJ, Roque GP. Factores de riesgo asociados com la tensión arterial en adolescentes. Rev Cubana Med Gen Integr. 2001;17(5):435-40.

27. Lamas JLT. O uso e abuso de drogas na adolescência. In: Anais do $3^{\circ}$ Congresso Paulista de Enfermagem Pediátrica e $5^{\mathrm{a}}$ Jornada de Enfermagem Pediátrica da UNICAMP; 2001; Campinas [CD-ROM]. Campinas: FCM/UNICAMP; 2001.

28. National High Blood Pressure Education Program Working Group on High Blood Pressure in Children and Adolescents. The fourth report on the diagnosis, evaluation, and treatment of high blood pressure in children and adolescents. Pediatrics. 2004;114(2 Suppl):555-76. 